\title{
A HOMOLOGICAL SELECTION THEOREM IMPLYING A DIVISION THEOREM FOR $Q$-MANIFOLDS
}

\author{
TARAS BANAKH \\ Instytut Matematyki, Akademia Świętokrzyska, Kielce, Poland, \\ Department of Mathematics, Ivan Franko Lviv National University, Lviv, Ukraine, \\ Nipissing University, North Bay, Canada \\ E-mail: tbanakh@yahoo.com \\ ROBERT CAUTY \\ Université Paris VI, France \\ E-mail: cauty@math.jussieu.fr
}

\begin{abstract}
We prove that a space $M$ with Disjoint Disk Property is a $Q$-manifold if and only if $M \times X$ is a $Q$-manifold for some $C$-space $X$. This implies that the product $M \times I^{2}$ of a space $M$ with the disk is a $Q$-manifold if and only if $M \times X$ is a $Q$-manifold for some $C$-space $X$. The proof of these theorems exploits the homological characterization of $Q$-manifolds due to Daverman and Walsh, combined with the existence of $G$-stable points in $C$-spaces. To establish the existence of such points we prove (and afterward apply) homological versions of the Brouwer Fixed Point Theorem and of Uspenskij's Selection Theorem.

It was noticed in $[\mathrm{BR}]$ that the famous Shchepin's Characterization Theorem for Tikhonov cube $[\mathrm{Sc}]$ implies a surprising
\end{abstract}

Theorem 1 (Division Theorem for Tikhonov cubes). If the product $X \times Y$ of two spaces is homeomorphic to a Tikhonov cube $[0,1]^{\tau}$ of uncountable weight $\tau$, then $X$ or $Y$ is homeomorphic to $[0,1]^{\tau}$.

This theorem has essentially non-metric nature and is not true for the Hilbert cube $Q=[0,1]^{\omega}$. A suitable counterexample can be found in [Sin].

ExAmPle 1 (Singh). There is a compact absolute retract $S$ such that $S \times[0,1]$ is homeomorphic to $Q$ but $S$ contains no closed ANR-subspace of dimension $\geq 2$.

Singh's space $S$ contains no topological copy of the 2-disk $I^{2}$ and hence does not possess the Disjoint Disks Property. We recall that a space $X$ has the Disjoint Disks

2000 Mathematics Subject Classification: 54C65, 55N10, 55M20, 54H25, 57N20, 57N75

The paper is in final form and no version of it will be published elsewhere. 
Property (briefly, DDP) if any two maps $f, g: I^{2} \rightarrow X$ from a 2-dimensional cube can be uniformly approximated by maps with disjoint images.

It turns out that the pathology appearing in Singh's example cannot occur among spaces with DDP. This fact was first noticed by Daverman and Walsh in [DW].

Theorem 2 (Daverman-Walsh). A space $M$ with DDP is a $Q$-manifold if and only if $M \times X$ is a $Q$-manifold for some finite-dimensional space $X$.

We recall that a topological space $X$ is a $Q$-manifold if it is paracompact and has an open cover by subsets homeomorphic to open subsets of the Hilbert cube $Q=[0,1]^{\omega}$.

In this paper we show that the finite-dimensionality of $X$ in the Daverman-Walsh Theorem can be weakened to the $C$-space property. We recall that a space $X$ is a $C$-space if for any countable sequence $\left(\mathcal{U}_{n}\right)_{n \in \omega}$ of open covers of $X$ there is a sequence $\left(\mathcal{V}_{n}\right)_{n \in \omega}$ such that $\bigcup_{n \in \omega} \mathcal{V}_{n}$ is a cover of $X$ and each $\mathcal{V}_{n}$ is a discrete family of open sets refining the cover $\mathcal{U}_{n}$.

The class of $C$-spaces includes all metrizable countable-dimensional spaces, i.e., spaces that can be written as countable unions of zero-dimensional subspaces, see [En, 6.3.8]. The famous Pol's example [En, 6.1.21] of a compact metrizable $C$-space that contains a strongly infinite-dimensional subspace shows that the class of $C$-spaces is not hereditary and is wider than the class of countable-dimensional spaces.

The following Division Theorem, generalizing Daverman-Walsh Theorem, is one of the principal results of this paper.

Theorem 3 (Division Theorem for $Q$-manifolds). A space $M$ with DDP is a $Q$-manifold if and only if the product $M \times X$ is a $Q$-manifold for some $C$-space $X$.

There is a version of this theorem not involving DDP. It generalizes Corollary 6.3 of $[\mathrm{DW}]$.

Theorem 4. If the product $M \times X$ of a space $M$ with a $C$-space $X$ is a $Q$-manifold, then the product $M \times I^{2}$ is a $Q$-manifold, too.

The proof of these theorems consists of two basic ingredients: the homological characterization of $Q$-manifolds due to Daverman and Walsh [DW] and Theorem 9 on existence of $G$-stable points in $C$-spaces. The proof of the latter theorem is not trivial and relies on homological versions of the Brouwer Fixed Point Theorem and Uspenskij's Selection Theorem [Us], see Theorems 7 and 8. The mentioned homological theorems seem to have independent value and may be helpful for some other applications.

1. Homological preliminaries. All topological spaces considered in the paper are Tikhonov. We use singular relative homology $H_{*}(X, A ; G)$ with coefficients in a nontrivial abelian group $G$. By $\widetilde{H}_{*}(X ; G)$ we denote the singular homology of $X$, reduced in dimension zero. If $G=\mathbb{Z}$, then we omit the symbol of the group and will write $H_{*}(X, A)$ in place of $H_{*}(X, A ; \mathbb{Z})$.

Among coefficient groups $G$, the most important for us are: the group $\mathbb{F}_{0}=\mathbb{Q}$ of rational numbers, and the cyclic groups $\mathbb{F}_{p}=\mathbb{Z} / p \mathbb{Z}$ of prime order $p$. Note that these groups carry the structure of a field, so we shall call them basic fields. Basic fields $\mathbb{F}_{p}$ 
are parametrized by numbers $p \in \Pi_{0}=\{0\} \cup \Pi$ where $\Pi$ stands for the set of all prime numbers.

In the following lemma we collect four homological results that will be applied later. The first three of them follow from the Universal Coefficients Formula [Hat, 3.A.3] and the last one from the Künneth formula (see Theorem 10 [Spa, §5.3]).

Lemma 1. Let $A \subset X, B \subset Y$ be closed subsets of topological spaces $X, Y$.

(1) If $H_{*}(X, A)=0$, then $H_{*}(X, A ; G)=0$ for any coefficient group $G$.

(2) If $H_{n}(X, A)$ contains an element of infinite order, then $H_{n}(X, A ; \mathbb{Q}) \neq 0$.

(3) If $H_{n}(X, A)$ contains an element of prime order $p$, then $H_{n+1}\left(X, A ; \mathbb{Z}_{p}\right) \neq 0$.

(4) If for some field $G$ the groups $H_{*}(X, X \backslash A ; G)$ and $H_{*}(Y, Y \backslash B ; G)$ are not trivial, then so is the group $H_{*}(X \times Y, X \times Y \backslash A \times B ; G)$.

Next, we recall the classical notion of a $Z$-set introduced by R.D. Anderson [An] and then define their homological counterparts called homological $Z$-sets (more detail information on such sets can be found in [BCK]). A closed subset $A$ of a space $X$ is called

- a $Z$-set if for every open set $U \subset X$ the relative homotopy groups $\pi_{k}(U, U \backslash A)$ are trivial for all $k$;

- a $G$-homological $Z$-set if for every open set $U \subset X$ the relative homology groups $H_{k}(U, U \backslash A ; G)$ are trivial for all $k$;

- a homological $Z$-set if it is a $\mathbb{Z}$-homological $Z$-set in $X$.

In $[\mathrm{DW}]$ homological $Z$-sets are referred to as closed sets with infinite codimension. Lemma 1(1) implies that each homological $Z$-set is a $G$-homological $Z$-set for any coefficient group $G$.

A point $x \in X$ is called a (homological) $Z$-point if the singleton $\{x\}$ is a (homological) $Z$-set in $X$. The Excision Axiom for singular homology [Hat, 2.20] implies that a point $x \in X$ is a $G$-homological $Z$-point if and only if $H_{k}(X, X \backslash\{x\} ; G)=0$ for all $k$.

In the following lemma (whose proof can be found in [DW] or $[\mathrm{BCK}]$ ) we collect some basic properties of homological $Z$-sets.

Lemma 2. Let $X$ be a Tikhonov space and $G$ be a non-trivial abelian group.

(1) If $A$ is a $G$-homological $Z$-set in $X$, then every closed subset $B$ of $A$ is a G-homological $Z$-set in $X$.

(2) For any $G$-homological $Z$-set in $X$ and any Tikhonov space $Y$ the product $A \times Y$ is a G-homological $Z$-set in $X \times Y$.

(3) A closed trt-dimensional subspace $A$ of $X$ is a $G$-homological $Z$-set in $X$ if and only if each point $a \in A$ is a $G$-homological Z-point in $X$.

A topological space $X$ is called trt-dimensional if $\operatorname{trt}(X) \leq \alpha$ for some ordinal $\alpha$, where $\operatorname{trt}(X)$ is the transfinite separation dimension introduced in $[\mathrm{ACP}]$ inductively:

- $\operatorname{trt}(X)=-1$ iff $X=\emptyset$; 
- $\operatorname{trt}(X) \leq \alpha$ for some ordinal $\alpha$ if each closed subspace $B \subset X$ containing more than one point can be separated by a closed subspace $P \subset B$ with $\operatorname{trt}(P)<\alpha$ (the latter means that $B \backslash P$ is disconnected).

It is clear that each finite-dimensional space is trt-dimensional. By [ACP, 4.7], each trt-dimensional compact space is a $C$-space and by [Rad], a locally compact space $X$ is trt-dimensional provided $X$ is $\sigma$-hereditarily disconnected in the sense that $X$ can be written as the countable union of hereditarily disconnected subspaces. In particular, each locally compact countable-dimensional space is trt-dimensional.

We define a point $x$ of a space $X$ to be $G$-stable if $x$ fails to be a $G$-homological $Z$-point in $X$ (equivalently, if $H_{*}(X, X \backslash\{x\} ; G) \neq 0$ ).

The last item of Lemma 2 implies

Corollary 1. Each trt-dimensional space $X$ contains a $G$-stable point $x_{G} \in X$ for every coefficient group $G$.

A $C$-space version of this corollary will be proved in Section 5 by more sophisticated methods. Now let us prove a result, useful for detecting homological $Z$-sets. We recall that by a basic field we understand either the field $\mathbb{F}_{0}=\mathbb{Q}$ or rational numbers or the finite field $\mathbb{F}_{p}=\mathbb{Z} / p \mathbb{Z}$ of a prime order $p$.

Lemma 3. Let $X$ be a locally path-connected space containing an $\mathbb{F}_{p}$-stable point $x_{p} \in X$ for every basic field $\mathbb{F}_{p}, p \in \Pi_{0}$. A subset $A$ of a topological space $M$ is a homological $Z$-set in $M$ if and only if for every $p \in \Pi_{0}$ the product $A \times\left\{x_{p}\right\}$ is a homological $Z$-set in $M \times X$.

Proof. The "only if" part of the lemma follows from Lemma 2. To prove the "if" part, assume that a closed subset $A$ of $M$ fails to be a homological $Z$-set in $M$, which means that $H_{*}(U, U \backslash A) \neq 0$ for some open set $U \subset M$. By Lemma $1(2,3), H_{*}\left(U, U \backslash A ; \mathbb{F}_{p}\right) \neq 0$ for some basic field $\mathbb{F}_{p}$. Since $H_{*}\left(X, X \backslash\left\{x_{p}\right\} ; \mathbb{F}_{p}\right) \neq 0$, we may apply Lemma $1(4)$ to conclude that $H_{*}\left(U \times X, U \times X \backslash A \times\left\{x_{p}\right\} ; \mathbb{F}_{p}\right) \neq 0$, which means that $A \times\left\{x_{p}\right\}$ is not an $\mathbb{F}_{p}$-homological $Z$-set in $M \times X$.

2. Homological characterization of $Q$-manifolds. In this section we survey some homological and product characterizations of $Q$-manifolds essentially due to Daverman and Walsh [DW].

TheOREm 5 ([DW, 6.1]). A space $X$ is a $Q$-manifold if and only if $X$ is a locally compact $A N R$ with $D D P$, containing a countable family $\mathcal{Z}$ of homological $Z$-sets such that each closed subset $A$ of $X$ missing $\bigcup \mathcal{Z}$ is a homological $Z$-set in $X$.

Theorem 6. A space $M$ with $D D P$ is a $Q$-manifold if and only if $M \times X$ is a $Q$-manifold for some space $X$ containing an $\mathbb{F}_{p}$-stable point $x_{p} \in X$ for every basic field $\mathbb{F}_{p}$.

Proof. This theorem was implicitly proved in Appendix of [DW] but not stated there as a separate result. So we give a proof for the convenience of the reader.

The "if" part of the theorem is trivial. To prove the "only if" part, assume that the product $M \times X$ is a $Q$-manifold for some space $X$ containing an $\mathbb{F}_{p}$-stable point $x_{p}$ for every basic field $\mathbb{F}_{p}$. It follows that both spaces $M$ and $X$ are locally compact ANR's. 
Since all points of the $Q$-manifold $M \times X$ are homological $Z$-points, we may apply Lemma 3 to conclude that all points of the space $M$ are homological $Z$-points.

The product $M \times X$, being a $Q$-manifold, contains a countable family $\left\{A_{n}: n \in \omega\right\}$ of finite-dimensional $Z$-sets such that each closed subset of $M \times X$ missing the union $\bigcup_{n} A_{n}$ is a homological $Z$-set in $M \times X$.

For every $n \in \omega$ and $p \in \Pi_{0}$ consider the closed finite-dimensional set $B_{n, p}=\{z \in M$ : $\left.\left(z, x_{p}\right) \in A_{n}\right\}$ in $M$. Since each point of $M$ is a homological $Z$-point, the sets $B_{n, p}$, being finite-dimensional (and thus trt-dimensional), are homological $Z$-sets in $M$, see Corollary 2.5 of $[\mathrm{DW}]$ (or Lemma 2(3) ). It remains to prove that each closed subset $F \subset X$ that misses the union $\bigcup_{n, p} B_{n, p}$ is a homological $Z$-set in $X$. Observe that for every $p \in \Pi_{0}$ the set $F \times\left\{x_{p}\right\}$ misses the union $\bigcup_{n \in \omega} A_{n}$ and thus is a homological $Z$-set in $M \times X$ by the choice of the family $\left\{A_{n}\right\}$. By Lemma $3, F$ is a homological $Z$-set in $M$.

Now it is legal to apply the Daverman-Walsh Theorem 5 to conclude that the space $M$ is a $Q$-manifold.

The following corollary of Theorem 6 generalizes Corollary 6.3 of [DW].

Corollary 2. For a space $M$ the following conditions are equivalent:

(1) $M \times I^{2}$ is a $Q$-manifold;

(2) $M \times X$ is a $Q$-manifold for some space $X$ containing an $\mathbb{F}_{p}$-stable point $x_{p}$ for every basic field $\mathbb{F}_{p}$.

Proof. The implication $(1) \Rightarrow(2)$ is trivial since the point $x_{0}=\left(\frac{1}{2}, \frac{1}{2}\right)$ of the square $I^{2}$ is $G$-stable for every group $G$ (because $H_{2}\left(I^{2}, I^{2} \backslash\left\{x_{0}\right\} ; G\right)=H_{1}\left(I^{2} \backslash\left\{x_{0}\right\} ; G\right)=G$ ).

$(2) \Rightarrow(1)$. Assume that $M \times X$ is a $Q$-manifold for some space $X$ containing an

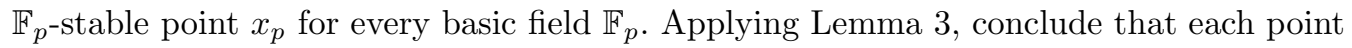
of the space $M$ is a homological $Z$-point. By Proposition 5.2 of [DW], the space $X \times I^{2}$ has DDP. Since $M \times I^{2} \times X$ is a $Q$-manifold, we may apply Theorem 6 , to conclude that $M \times I^{2}$ is a $Q$-manifold.

Combining the preceding results with Corollary 1 we obtain "trt-dimensional" versions of Theorems 3 and 4:

Proposition 1. A space $M$ with DDP is a $Q$-manifold if and only if the product $M \times X$ is a $Q$-manifold for trt-dimensional space $X$.

Proposition 2. If the product $M \times X$ of a space $M$ with a trt-dimensional space $X$ is a Q-manifold, then the product $M \times I^{2}$ is a $Q$-manifold, too.

Theorems 3 and 4 will follow in the same manner as soon as we prove that each locally compact locally contractible $C$-space $X$ contains an $\mathbb{F}_{p}$-stable point $x_{p}$ for every basic field $\mathbb{F}_{p}$. For this we need to elaborate some tools like homological versions of the Brouwer Fixed Point Theorem and of the Uspenskij Selection Theorem [Us].

3. A Homological Fixed Point Theorem. In this section we prove a homological version of the classical Brouwer Fixed Point Theorem asserting that each continuous map $f: X \rightarrow X$ on a compact absolute retract $X$ has a fixed point. The homological version of this result guarantees the existence of a fixed point for chain morphisms between 
complexes of singular chains on $X$ and by its spirit is close to the classical Lefschetz-Hopf Fixed Point Theorem, see [Bre, 23.4].

First we recall some definitions. Throughout this section, $G$ is a field. For a subset $A$ of a topological space $X$ and a cover $\mathcal{U}$ of $X$ let $\mathcal{S} t(A, \mathcal{U})=\bigcup\{U \in \mathcal{U}: U \cap A \neq \emptyset\}$ be the star of $A$ with respect to the cover $\mathcal{U}$. Also we shall write $A \prec \mathcal{U}$ if $A \subset U$ for some $U \in \mathcal{U}$.

For a space $X$ by $S(X ; G)$ we denote the complex of singular chains of the space $X$. So $S(X ; G)=\bigoplus_{k \geq 0} S_{k}(X ; G)$ is the direct sum of free modules $S_{k}(X ; R)$ over $G$ generated by singular $k$-simplexes in $X$. The modules $S_{k}(X ; G)$ are linked via the boundary homomorphisms $\partial: S_{k}(X ; G) \rightarrow S_{k-1}(X ; G)$ where $S_{-1}(X ; G)=G$.

By $\Delta^{k}=\left\{\left(t_{0}, \ldots, t_{k}\right) \in[0,1]^{k+1}: t_{0}+\ldots+t_{k}=1\right\}$ we denote the standard $k$-simplex. For a singular $k$-simplex $\sigma: \Delta^{k} \rightarrow X$ by $\|\sigma\|=\sigma\left(\Delta^{k}\right)$ we denote the carrier of $\sigma$ and for a $k$-chain $c \in S_{k}(X ; G)$ we put $\|c\|=\bigcup_{i}\left\|\sigma_{i}\right\|$, where $c=\sum_{i} g_{i} \sigma_{i}$ is the irreducible representation of $c$ as a linear combination of singular $k$-simplexes.

For a cover $\mathcal{U}$ of $X$ let $S(X, \mathcal{U} ; G)$ stand for the submodule of $S(X ; G)$, generated by singular simplexes whose supports lie in elements of $\mathcal{U}$.

A homomorphism $\varphi: S(X, \mathcal{U} ; G) \rightarrow S(Y ; G)$ is called a chain morphism if $\partial \circ \varphi=\varphi \circ \partial$ and $\varphi\left(S_{k}(X, \mathcal{U} ; G)\right) \subset S_{k}(Y ; G)$ for all $k \in \omega$. Each continuous map $f: X \rightarrow Y$ induces a chain morphism $f_{\#}: S(X, \mathcal{U} ; G) \rightarrow S(Y ; G)$ assigning to a singular simplex $\sigma: \Delta^{k} \rightarrow X$ the simplex $f \circ \sigma: \Delta^{k} \rightarrow Y$.

A point $x \in X$ is called a fixed point of a chain morphism $\varphi: S(X, \mathcal{U} ; G) \rightarrow S(X ; G)$ if for any neighborhood $V \prec \mathcal{U}$ of $x$ there is a chain $c \in S(V ; G)$ such that $\|\varphi(c)\| \cap V \neq \emptyset$.

Observe that the set of fixed points of a continuous map $f: X \rightarrow X$ coincide with the set of fixed points of the induced chain morphism $f_{\#}: S(X, \mathcal{U} ; G) \rightarrow S(X ; G)$. So, detecting fixed points of chain morphisms can help in detecting fixed points of continuous maps.

The principal result of this section is the following homological version of the Brouwer Fixed Point Theorem. For the field $G=\mathbb{Q}$ of rational numbers a much more general result (treating compact maps of so-called algebraic ANR's) was proved in Theorem 5 of [Ca]. However in the framework of usual AR's it has a simpler proof, which we include here for the convenience of the reader.

Theorem 7 (A Homological Fixed Point Theorem). Let $G$ be a field and $\mathcal{U}$ be an open cover of a compact absolute retract $X$. Any chain morphism $\varphi: S(X, \mathcal{U} ; G) \rightarrow S(X ; G)$ has a fixed point $x \in X$.

Proof. First, we reduce the problem to the case $X=I^{n}$ for a suitable $n \in \mathbb{N}$. Suppose that some chain morphism $\varphi: S(X, \mathcal{U} ; G) \rightarrow S(X ; G)$ has no fixed point.

Embed $X$ into the Hilbert cube $Q$ and take any retraction $r: Q \rightarrow X$. Since the chain morphism $\varphi$ fails to have a fixed point, then so does the morphism $\varphi \circ r_{\#}$ : $S\left(Q, r^{-1}(\mathcal{U}) ; G\right) \rightarrow S(X ; G) \subset S(Q ; G)$. Therefore, we lose no generality assuming that $X=Q$.

The absence of a fixed point for $\varphi$ implies the existence of an open cover $\mathcal{V}$ of $X$ inscribed into $\mathcal{U}$ and such that $\|\varphi(c)\| \cap \mathcal{S} t(V, \mathcal{V})=\emptyset$ for every $V \in \mathcal{V}$ and every singular chain $c \in S(V ; G)$. We can assume that all sets $V \in \mathcal{V}$ are of cylindrical form, that is 
$V=p^{-1}(p(V))$, where $p: Q \rightarrow I^{n}$ is the natural projection of $Q=[0,1]^{\omega}$ onto the $n$ dimensional cube $I^{n}=\left\{\left(t_{i}\right) \in Q: t_{i}=0\right.$ for all $\left.i \geq n\right\}$ for some $n$. Denoting by $i: I^{n} \rightarrow Q$ the identity embedding, we see that the morphism $p_{\#} \circ \varphi \circ i_{\#}: S\left(I^{n}, p(\mathcal{V}) ; G\right) \rightarrow S\left(I^{n} ; G\right)$ also has no fixed point. So, we lose no generality assuming that $X=I^{n}$ for some $n$.

Let $\mathcal{T}$ be a triangulation of $I^{n}$ so fine that the closed star $\overline{\mathcal{S t}}(\sigma)$ of every simplex $\sigma \in \mathcal{T}$ lies in some set $V \in \mathcal{V}$. Denote by $\mathcal{W}$ the cover of $I^{n}$ by open stars of vertices of the triangulation $\mathcal{T}$. Let $\mathcal{T}^{\prime}$ be the barycentric subdivision of $\mathcal{T}$ and let $C\left(\mathcal{T}^{\prime} ; G\right)$ denote the complex of oriented simplicial chains of $\mathcal{T}^{\prime}$ with coefficients in the field $G$. For every simplex $\sigma$ of $\mathcal{T}^{\prime}$ fix a corresponding generator of $C\left(\mathcal{T}^{\prime} ; G\right)$ and denote it by the same symbol $\sigma$. By $\|\sigma\| \subset I^{n}$ we denote the geometric realization of a simplex $\sigma$ and for a simplicial chain $c \in C\left(\mathcal{T}^{\prime} ; G\right)$ we put $\|c\|=\bigcup_{i}\left\|\sigma_{i}\right\|$ where $c=\sum_{i} g_{i} \sigma_{i}$ is an irreducible representation of $c$ as a linear combination of oriented simplexes $\sigma_{i}$ with non-zero coefficients.

Using the acyclicity of geometric simplexes, by induction on dimension one can construct a chain morphism $\eta: C\left(\mathcal{T}^{\prime} ; G\right) \rightarrow S\left(I^{n} ; G\right)$ such that $\|\eta(\sigma)\| \subset\|\sigma\|$ for all simplexes $\sigma$ of $\mathcal{T}^{\prime}$. According to [Spa, 4.4.14], there is an operator of subdivision $\mathrm{Sd}: S\left(I^{n} ; G\right) \rightarrow$ $S\left(I^{n}, \mathcal{W} ; G\right)$ such that $\|\operatorname{Sd}(c)\| \subset\|c\|$ for any singular chain $c \in S\left(I^{n} ; G\right)$.

Next, we are going to construct a chain morphism $\psi: S\left(I^{n}, \mathcal{W} ; G\right) \rightarrow C\left(\mathcal{T}^{\prime} ; G\right)$ such that $\|\psi(c)\| \subset \mathcal{S} t(\|c\|, \mathcal{W})$ for every singular chain $c \in S\left(I^{m}, \mathcal{W} ; G\right)$. The construction of $\psi$ is inductive and relies on the notion of the traverse $\operatorname{Tr}(\sigma)$ of a simplex $\sigma$ of $\mathcal{T}$.

First some notation. We write $\tau \subseteq \sigma$ if a simplex $\tau$ is a face of a simplex $\sigma$ and $\tau \subsetneq \sigma$ if $\tau$ is a proper face of $\sigma$. By $b_{\sigma}$ we denote the barycenter of a simplex $\sigma$.

The traverse $\operatorname{Tr}(\sigma)$ of a simplex $\sigma$ of the triangulation $\mathcal{T}$ is the subcomplex of the barycentric subdivision $\mathcal{T}^{\prime}$ formed by geometric simplexes with vertices $b_{\sigma_{0}}, b_{\sigma_{1}}, \ldots, b_{\sigma_{n}}$ where $\sigma \subseteq \sigma_{0} \subsetneq \cdots \subsetneq \sigma_{n}$ are simplexes of $\mathcal{T}$. It is well known (and easy to see) that the traverse $\operatorname{Tr}(\sigma)$, being a cone with vertex $b_{\sigma}$, is a contractible subcomplex of $\mathcal{T}^{\prime}$. It is also clear that $\operatorname{Tr}(\sigma) \subset \mathcal{S} t(\|\sigma\|, \mathcal{W})$ and $\operatorname{Tr}(\tau) \supset \operatorname{Tr}(\sigma)$ if $\tau$ is a face of $\sigma$.

For every subset $A \subset I^{n}$ with $A \prec \mathcal{W}$ denote by $s(A)$ the simplex of $\mathcal{T}$ with the vertices $v$ such that $A \subset \mathcal{S t}(v, \mathcal{W})$. Observe that for subsets $A \subset B$ of $I^{n}$ with $B \prec \mathcal{W}$ we get $s(A) \supset s(B)$ and $\operatorname{Tr}(s(A)) \subset \operatorname{Tr}(s(B))$.

By induction we shall construct a chain morphism $\psi: S\left(I^{n}, \mathcal{W} ; G\right) \rightarrow C\left(\mathcal{T}^{\prime} ; G\right)$ such that $\|\psi(\sigma)\| \subset \operatorname{Tr}(s(\|\sigma\|))$ for every simplex $\sigma \in S\left(I^{n}, \mathcal{W} ; G\right)$. For a 0 -dimensional singular simplex $\sigma$ we let $\psi(\sigma)$ be the barycenter $b_{s(\|\sigma\|)}$ of the simplex $s(\|\sigma\|)$. Assuming that for some $k \geq 0$ the chain morphism $\psi$ is defined on the space $S_{k}\left(I^{n}, \mathcal{W} ; G\right)$, take any singular $(k+1)$-simplex $\sigma \in S\left(I^{n}, \mathcal{W} ; G\right)$ and consider the chain $\partial \sigma$. By the inductive assumption, for every face $\tau$ of $\sigma$ we get $\|\psi(\tau)\| \subset \operatorname{Tr}(s(\|\tau\|)) \subset \operatorname{Tr}(s(\|\sigma\|))$. Then $\|\psi(\partial \sigma)\| \subset \operatorname{Tr}(s(\|\sigma\|))$ and the contractibility of the traverse $\operatorname{Tr}(s(\|\sigma\|))$ will help us to find a chain $c \in C_{k+1}\left(\mathcal{T}^{\prime} ; G\right)$ with $\|c\| \subset \operatorname{Tr}(s(\|\sigma\|))$ and $\partial c=\psi(\partial \sigma)$. Letting $\psi(\sigma)=c$ we define the morphism $\psi$ on each $(k+1)$-simplex $\sigma \in S\left(I^{n}, \mathcal{W} ; G\right)$, and this morphism can be extended onto $S_{k+1}(X, \mathcal{W} ; G)$ by linearity.

It follows from the construction that for any singular simplex $\sigma \in S\left(I^{n}, \mathcal{W} ; G\right)$ we get $\|\psi(\sigma)\| \subset \operatorname{Tr}(s(\|\sigma\|)) \subset \mathcal{S} t(s(\|\sigma\|), \mathcal{W}) \subset \mathcal{S} t(\|\sigma\|, \mathcal{W})$. The linearity of $\psi$ implies that $\|\psi(c)\| \subset \mathcal{S} t(\|c\|, \mathcal{W})$ for any chain $c \in S\left(I^{n}, \mathcal{W} ; G\right)$. 
Having constructed the morphism $\psi$, consider the chain morphism

$$
\xi=\psi \circ \operatorname{Sd} \circ \varphi \circ \eta: C\left(\mathcal{T}^{\prime} ; G\right) \rightarrow C\left(\mathcal{T}^{\prime} ; G\right) .
$$

We claim that for every $k \geq 0$ the $k$ th component $\xi_{k}: C_{k}\left(\mathcal{T}^{\prime} ; G\right) \rightarrow C_{k}\left(\mathcal{T}^{\prime} ; G\right)$ of this morphism has zero trace; more precisely, the matrix of $\xi_{k}$ in the canonical base of $C\left(\mathcal{T}^{\prime} ; G\right)$ has zero trace. The canonical base of $C\left(\mathcal{T}^{\prime} ; G\right)$ consists of oriented simplexes $\sigma$ of the triangulation $\mathcal{T}^{\prime}$. For any such a simplex $\sigma$ take any $V \in \mathcal{V}$ with $\|\sigma\| \subset V$ and observe that $\|\eta(\sigma)\| \subset\|\sigma\| \subset V$ and hence $\|\varphi(\eta(\sigma))\| \cap \mathcal{S} t(V, \mathcal{V})=\emptyset$. Then

$$
\|\operatorname{Sd} \circ \varphi \circ \eta(\sigma)\| \subset\|\varphi \circ \eta(\sigma)\| \subset I^{n} \backslash \mathcal{S} t(V, \mathcal{V})
$$

and

$$
\begin{aligned}
\|\xi(\sigma)\|=\|\psi \circ \operatorname{Sd} \circ \varphi \circ \eta(\sigma)\| & \subset \mathcal{S} t(\|\operatorname{Sd} \circ \varphi \circ \eta(\sigma)\|, \mathcal{W}) \\
& \subset \mathcal{S} t\left(I^{n} \backslash \mathcal{S} t(V, \mathcal{V}), \mathcal{W}\right) \subset I^{n} \backslash V \subset I^{n} \backslash\|\sigma\|,
\end{aligned}
$$

which means that for the representation $\xi(\sigma)=\sum_{\tau} g_{\tau} \cdot \tau$ of $\xi(\sigma)$ as a linear combination of $k$-simplexes, the coefficient $g_{\sigma}=0$. Therefore the matrix of the transformation $\xi_{k}$ has zero diagonal and zero $\operatorname{trace} \operatorname{tr}\left(\xi_{k}\right)=0$.

Then the Lefschetz number

$$
\Lambda(\xi)=\sum_{k \geq 0}(-1)^{k} \operatorname{tr}\left(\xi_{k}\right)
$$

of the chain morphism $\xi$ equals zero. On the other hand, the Hopf Trace Formula [Bre, 2.3.2] implies that $\Lambda(\xi)=\Lambda\left(\xi_{*}\right)$, where $\xi_{*}: H_{*}\left(I^{n} ; G\right) \rightarrow H_{*}\left(I^{n} ; G\right)$ is the induced homomorphism in homologies. All homological groups of $I^{n}$, except for $H_{0}\left(I^{n} ; G\right)=G$ are trivial, which implies that $1=\Lambda\left(\xi_{*}\right)=\Lambda(\xi)=0$ and this is a contradiction, completing the proof of the theorem.

4. A homological version of Uspenskij's Selection Theorem. In this section we prove another important ingredient for the proof of Theorem 9-a homological version of Uspenskij's Selection Theorem [Us]. We recall that Uspenskij's Theorem guarantees the existence of a continuous selection for each strongly lower semicontinuous multivalued function $\Phi: X \Rightarrow Y$ defined on a paracompact $C$-space $X$ and having aspherical values.

A multivalued function $\Phi: X \Rightarrow Y$ is called strongly lower semicontinuous if for each compact subset $K \subset Y$ the set $\{x \in X: K \subset \Phi(x)\}$ is open in $X$.

A space $X$ is aspherical if its homotopy groups all are trivial. A homological version of Uspenskij's Selection Theorem treats multivalued functions having $G$-acyclic values, where a space $X$ is defined to be $G$-acyclic if its singular homology $\widetilde{H}_{*}(X ; G)$ (reduced in dimension zero) with the coefficient group $G$ is trivial.

TheOREM 8. Let $G$ be a ring with unit, $X$ be a paracompact $C$-space, $Y$ be a topological space, and $\Phi: X \Rightarrow Y$ be a strongly lower semicontinuous multivalued function having $G$-acyclic values $\Phi(x)$ for all $x \in X$. Then there are an open cover $\mathcal{U}$ of $X$ and a chain morphism $\varphi: S(X, \mathcal{U} ; G) \rightarrow S(Y ; G)$ such that $\varphi(S(U ; G)) \subset S(\Phi(x) ; G)$ for every $U \in \mathcal{U}$ and every point $x \in U$.

Proof. We shall construct two sequences of locally finite open covers of $X, \mathcal{V}_{n}=\left\{V_{\alpha}\right.$ : $\left.\alpha \in A_{n}\right\}$ and $\mathcal{W}_{n}=\left\{W_{\alpha}: \alpha \in A_{n}\right\}$ of $X$ so that 
(1) $\bar{W}_{\alpha} \subset V_{\alpha}$ for all $\alpha$.

The index sets $A_{n}$ will be taken pairwise disjoint and we shall construct an increasing sequence $K_{1} \subset K_{2} \subset \ldots$ of simplicial complexes and chain morphisms $\mu_{n}: C\left(K_{n} ; G\right) \rightarrow$ $S(Y ; G)$ satisfying

(2) $\mu_{n} \mid C\left(K_{n-1} ; G\right)=\mu_{n-1}$.

To every index $\alpha \in A_{n}$ we shall assign a finite contractible subcomplex $L_{\alpha}$ of $K_{n}$ in such a way that the compact set

$$
C_{\alpha}=\bigcup_{\sigma \subset L_{\alpha}}\left\|\mu_{n}(\sigma)\right\| .
$$

will satisfy the inclusion

(3) $C_{\alpha} \subset \Phi(x)$ for all $x \in V_{\alpha}$.

For every $x \in X$, fix a point $y_{x} \in \Phi(x)$ and consider the set $O_{x}=\left\{x^{\prime} \in X: y_{x} \in \Phi\left(x^{\prime}\right)\right\}$ which is an open neighborhood of $x$ because $\Phi$ is strongly lower semicontinuous. Let $\mathcal{V}_{1}=\left\{V_{\alpha}: \alpha \in A_{1}\right\}$ be an open locally finite cover of $X$ inscribed into $\left\{O_{x}: x \in X\right\}$, and let $\mathcal{W}=\left\{W_{\alpha}: \alpha \in A_{1}\right\}$ be a locally finite open cover satisfying (1). Let $K_{1}$ be the zero-dimensional complex having $A_{1}$ for the set of vertices. For every $\alpha \in A_{1}$ let $L_{\alpha}=\{\alpha\}$ and choose $x_{\alpha} \in X$ so that $O_{x_{\alpha}}$ contains $V_{\alpha}$. Let $\mu_{1}: C\left(K_{1} ; G\right) \rightarrow S(Y ; G)$ be the chain morphism assigning to the generator corresponding to $\alpha$ the 0 -simplex $y_{x_{\alpha}}$. Then the set $C_{\alpha}=\left\{y_{x_{\alpha}}\right\}$ satisfies (3) by the definition of $O_{x_{\alpha}}$.

Assume that for some $n \geq 1$, the covers $\mathcal{V}_{n}$ and $\mathcal{W}_{n}$ have been constructed. For every $x \in X$ let $P_{x}$ be an open neighborhood of $x$ meeting only finitely many sets of the locally finite cover $\bigcup_{p \leq n} \mathcal{V}_{p}$ and such that $P_{x} \subset V_{\alpha}$ for every $\alpha \in A_{p}$ with $P_{x} \cap \bar{W}_{\alpha} \neq \emptyset$. Let $J(x)$ be the set of all $\alpha \in A_{1} \cup \ldots \cup A_{n}$ such that $P_{x} \subset V_{\alpha}$ and let $D_{x}=\bigcup\left\{C_{\alpha}\right.$ : $\alpha \in J(x)\}$. The set $J(x)$ is finite, so $D_{x}$ is compact, and the condition (3) guarantees that $D_{x} \subset \Phi(x)$. Let $M_{x}=\bigcup\left\{L_{\alpha}: \alpha \in J(x)\right\}$; this is a finite subcomplex of $K_{n}$, and the definition of $C_{\alpha}$ ensures that $\mu_{n}\left(C\left(M_{x} ; G\right)\right) \subset S\left(D_{x} ; G\right) \subset S(\Phi(x) ; G)$. The $G$-acyclicity of $\Phi(x)$ guarantees that, for the cone $L_{x}$ with base $M_{x}$ and vertex $v_{x} \notin M_{x}$, there is a chain morphism $\mu_{x}: C\left(L_{x} ; G\right) \rightarrow S(\Phi(x) ; G)$ extending $\mu_{n} \mid C\left(M_{x} ; G\right)$. Then $C_{x}=\bigcup_{\sigma \subset L_{x}}\left\|\mu_{x}(\sigma)\right\|$ is a compact set containing $D_{x}$ and lying in $\Phi(x)$. Let $O_{x}^{n}=\left\{x^{\prime} \in\right.$ $\left.P_{x}: C_{x} \subset \Phi\left(x^{\prime}\right)\right\}$. Since $\Phi$ is strongly lower semicontinuous, $O_{x}^{n}$ is an open neighborhood of $x$.

Let $\mathcal{V}_{n+1}=\left\{V_{\alpha}: \alpha \in A_{n+1}\right\}$ be a locally finite open cover of $X$ inscribed into $\left\{O_{x}^{n}: x \in X\right\}$, and let $\mathcal{W}_{n+1}=\left\{W_{\alpha}: \alpha \in A_{n+1}\right\}$ be a locally finite open cover of $X$, satisfying (1). For every $\alpha \in A_{n+1}$, choose a point $x_{\alpha} \in X$ such that $O_{x_{\alpha}}^{n}$ contains $V_{\alpha}$, and let $L_{\alpha}$ be the cone with base $M_{x_{\alpha}}$ and vertex $\alpha$. Put $K_{n+1}=K_{n} \cup \bigcup_{\alpha \in A_{n+1}} L_{\alpha}$. Let $\psi_{\alpha}$ be the simplicial isomorphism of $L_{\alpha}$ onto $L_{x_{\alpha}}$ which is identity on $M_{x_{\alpha}}$ and maps $\alpha$ onto $v_{x_{\alpha}}$. Define the chain morphism $\mu_{n+1}: C\left(K_{n+1} ; G\right) \rightarrow S(Y ; G)$ by $\mu_{n+1} \mid C\left(K_{n} ; G\right)=\mu_{n}$ and $\mu_{n+1} \mid C\left(L_{\alpha} ; G\right)=\mu_{x_{\alpha}} \circ\left(\psi_{\alpha}\right) \#$ for all $\alpha \in A_{n+1}$. Then $C_{\alpha}=C_{x_{\alpha}}$, and the definition of $O_{x_{\alpha}}^{n}$ guarantees that (3) holds. Moreover, since $O_{x_{\alpha}}^{n} \subset P_{x_{\alpha}}$, the definition of $P_{x}$ guarantees that

(4) If $\alpha^{\prime} \in A_{1} \cup \ldots \cup A_{n}$ and $\alpha \in A_{n+1}$ satisfy $W_{\alpha} \cap W_{\alpha^{\prime}} \neq \emptyset$, then $W_{\alpha} \subset V_{\alpha^{\prime}}$, and thus $L_{\alpha^{\prime}} \subset L_{\alpha}$. 
Let $K=\bigcup_{n=1}^{\infty} K_{n}$. Since $X$ is a $C$-space, there are disjoint families $\mathcal{U}_{n}=$ $\left\{U_{\lambda}: \lambda \in \Lambda_{n}\right\}, n \geq 1$, of open sets in $X$ such that every $\mathcal{U}_{n}$ refines $\mathcal{W}_{n}$ and $\mathcal{U}=\bigcup \mathcal{U}_{n}$ covers $X$. We suppose that the index sets $\Lambda_{n}$ are pairwise disjoint and put $\Lambda=\bigcup_{n=1}^{\infty} \Lambda_{n}$, $\Lambda_{\leq n}=\Lambda_{1} \cup \ldots \cup \Lambda_{n}$, and

$$
S_{n}=\sum_{\lambda \in \Lambda_{\leq n}} S\left(U_{\lambda} ; G\right) .
$$

The sets $S_{n}$ form an increasing sequence of subcomplexes of $S(X ; G)$ with union equal to $S(X, \mathcal{U} ; G)$. For every $\lambda \in \Lambda_{n}$, select an $\alpha_{\lambda} \in A_{n}$ such that $U_{\lambda} \subset W_{\alpha_{\lambda}}$. We are going to construct a chain morphism $\nu: S(X, \mathcal{U} ; G) \rightarrow C(K ; G)$ such that

(5) $\nu\left(S\left(U_{\lambda} ; G\right)\right) \subset C\left(L_{\alpha_{\lambda}} ; G\right)$ for all $\lambda \in \Lambda$.

Since $L_{\alpha_{\lambda}}$ is contractible, for every $\lambda \in \Lambda_{1}$ there is a chain morphism $\nu_{\lambda}: S\left(U_{\lambda} ; G\right) \rightarrow$ $C\left(L_{\alpha_{\lambda}} ; G\right)$. Since the family $U_{\lambda}, \lambda \in \Lambda_{1}$, is disjoint, $S_{1}$ is a direct sum of $S\left(U_{\lambda} ; G\right)$ for $\lambda \in \Lambda_{1}$, so we may define the restriction $\nu_{1}$ of $\nu$ to $S_{1}$ letting $\nu_{1} \mid S\left(U_{\lambda} ; G\right)=\nu_{\lambda}$ for all $\lambda \in \Lambda_{1}$.

Suppose that for some $n \geq 1$ the restriction $\nu_{n}$ of $\nu$ to $S_{n}$ has been constructed. If $\lambda_{1}, \lambda_{2}$ are two distinct elements of $\Lambda_{n+1}$, then $S\left(U_{\lambda_{1}} ; G\right) \cap S\left(U_{\lambda_{2}} ; G\right)=\{0\}$. So, to extend $\nu_{n}$ to $S_{n+1}$, it suffices to construct extensions $\nu_{\lambda}$ of $\nu_{n} \mid S\left(U_{\lambda} ; G\right) \cap S_{n}$ onto $S\left(U_{\lambda} ; G\right)$ for every $\lambda \in \Lambda_{n+1}$. If $\lambda^{\prime} \in \Lambda_{\leq n}$ satisfies $U_{\lambda} \cap U_{\lambda^{\prime}} \neq \emptyset$, then $W_{\alpha_{\lambda}} \cap W_{\alpha_{\lambda^{\prime}}} \neq \emptyset$, so $L_{\alpha_{\lambda^{\prime}}} \subset L_{\alpha_{\lambda}}$ according to (4). The condition (5) implies $\nu_{n}\left(S\left(U_{\lambda} ; G\right) \cap S_{n}\right) \subset C\left(L_{\alpha_{\lambda}} ; G\right)$, and the $G$-acyclicity of $L_{\alpha_{\lambda}}$ ensures the existence of the desired extension $\nu_{\lambda}$.

Let $\varphi=\mu \circ \nu: S(X, \mathcal{U} ; G) \rightarrow S(Y ; G)$. Using (5) and the definition of $C_{\alpha}$, we get, for every $\lambda \in \Lambda$,

$$
\varphi\left(S\left(U_{\lambda} ; G\right)\right) \subset \mu\left(C\left(L_{\alpha_{\lambda}} ; G\right)\right) \subset S\left(C_{\alpha_{\lambda}} ; G\right) .
$$

Since $U_{\lambda} \subset W_{\alpha_{\lambda}} \subset V_{\alpha_{\lambda}}$, (3) implies that $C_{\alpha_{\lambda}} \subset \Phi(x)$ for every $x \in U_{\lambda}$, which completes the proof of the theorem.

5. $G$-stable points in $C$-spaces. Now we are able to prove a promised theorem, which being combined with Theorem 6 and Corollary 2, implies Theorems 3 and 4 announced in the Introduction. For the ring $G=\mathbb{Z}$ this theorem was proved in [BCK].

THEOREM 9. Each metrizable locally compact locally contractible $C$-space $X$ contains a $G$-stable point $x_{G} \in X$ for every field $G$.

Proof. By Gresham's Theorem [Gre], $X$, being a locally contractible $C$-space, is an ANR. Then Edwards' ANR-Theorem [Chap, 44.1] guarantees that the product $M=X \times Q$ is a $Q$-manifold. Take an open subset $W \subset M$ such that the closure $\bar{W}$ of $W$ in $M$ is homeomorphic to the Hilbert cube and the remainder $\bar{W} \backslash W$ is a homotopical $Z$-set in $\bar{W}$. Let pr : $\bar{W} \rightarrow X$ stand for the natural projection.

To derive a contradiction, assume that for some field $G$ the space $X$ contains no $G$-stable point; in other terms, each point $x$ of $X$ is a $G$-homological $Z$-point. Then Lemma 2 ensures that the product $\{x\} \times Q$ is a $G$-homological $Z$-set in $M \times X$, which guarantees that $H_{*}(W, W \backslash(\{x\} \times Q) ; G)=0$. The exact sequence of the pair $(W, W \backslash(\{x\} \times Q))=\left(W, W \backslash \operatorname{pr}^{-1}(x)\right)$ implies that

$$
\widetilde{H}_{*}\left(W \backslash \mathrm{pr}^{-1}(x) ; G\right)=H_{*}\left(W, W \backslash \mathrm{pr}^{-1}(x) ; G\right)=0 .
$$


Since the remainder $\bar{W} \backslash W$ is a homotopical $Z$-set in $\bar{W}$, the space $\bar{W} \backslash \operatorname{pr}^{-1}(x)$ is homotopy equivalent to $W \backslash \operatorname{pr}^{-1}(x)$ and hence $\widetilde{H}_{*}\left(\bar{W} \backslash \operatorname{pr}^{-1}(x) ; G\right)=0$, which means that the multivalued function

$$
\Phi: X \Rightarrow \bar{W}, \quad \Phi: x \mapsto \bar{W} \backslash \operatorname{pr}^{-1}(x)
$$

has $G$-acyclic values.

Observe that for every compact subset $K \subset \bar{W}$ the set

$$
\{x \in X: K \subset \Phi(x)\}=X \backslash \operatorname{pr}(K)
$$

is open in $X$, which means that the multivalued function $\Phi: X \Rightarrow \bar{W}$ is strongly lower semicontinuous.

Now it is legal to apply Theorem 8 to find an open cover $\mathcal{U}$ on $X$ and a chain morphism $\varphi: S(X, \mathcal{U} ; G) \rightarrow S(\bar{W} ; G)$ such that $\varphi(S(U ; G)) \subset S(\Phi(x) ; G)$ for every $U \in \mathcal{U}$ and every point $x \in U$.

Consider the open cover $\operatorname{pr}^{-1}(\mathcal{U})=\left\{\operatorname{pr}^{-1}(U): U \in \mathcal{U}\right\}$ of a Hilbert cube $\bar{W}$, and let $\operatorname{pr}_{*}: S\left(\bar{W}, \operatorname{pr}^{-1}(\mathcal{U}) ; G\right) \rightarrow S(X, \mathcal{U} ; G)$ be the chain morphism induced by the projection pr $: \bar{W} \rightarrow X$.

By the Homological Fixed Point Theorem 7, for the chain morphism

$$
\varphi \circ \operatorname{pr}_{*}: S\left(\bar{W}, \operatorname{pr}^{-1}(\mathcal{U}) ; G\right) \rightarrow S(\bar{W} ; G)
$$

there are a set $U \in \mathcal{U}$ and a chain $c \in S\left(\mathrm{pr}^{-1}(U) ; G\right)$ such that $\left\|\varphi \circ \mathrm{pr}_{*}(c)\right\| \cap \mathrm{pr}^{-1}(U)$ contains some point $(x, q)$. Since $\operatorname{pr}_{*}(c) \in S(U ; G)$, the choice of the morphism $\varphi$ guarantees that $\varphi\left(\operatorname{pr}_{*}(c)\right) \in S(\Phi(x) ; G)$ and hence $\left\|\varphi \circ \operatorname{pr}_{*}(c)\right\| \subset \Phi(x)=\bar{W} \backslash \operatorname{pr}^{-1}(x) \subset \bar{W} \backslash\{(x, q)\}$, which contradicts the choice of the point $(x, q)$.

6. Open problems. Theorems 3 and 4 would follow from (more simple) Propositions 1 and 2 if the answer to the (first part of the) following question were affirmative.

Problem 1. Is each compact $C$-space trt-dimensional? Is each compact trt-dimensional space $\sigma$-hereditarily disconnected?

Our other problem asks if Theorem 9 is true for weakly infinite-dimensional compacta.

Problem 2. Let $X$ be a compact weakly infinite-dimensional ANR-space. Does $X$ contain a $G$-stable point $x_{G}$ for every (basic) field $G$ ?

Acknowledgements. The authors express their sincere thanks to Roman Pol and Taras Radul for valuable remarks concerning the content and problems from the (previous version of) the paper.

\section{References}

[An] R. D. Anderson, On topological infinite deficiency, Michigan Math. J. 14 (1967), 365-383.

[ACP] F. G. Arenas, V. A. Chatyrko, M. L. Puertas, Transfinite extension of Steinke's dimension, Acta Math. Hungar. 88 (2000), 105-112.

[BCK] T. Banakh, R. Cauty, A. Karassev, On homotopical and homological $Z_{n}$-sets, preprint. 
[BR] T. Banakh, D. Repovš, Division and $k$-th root theorems for $Q$-manifolds, Sci. China Ser. A, to appear.

[Bre] G. E. Bredon, Topology and Geometry, Grad. Texts in Math. 139, Springer, New York 1993.

[Ca] R. Cauty, Rétractes absolus de voisinage algébriques, Serdica Math. J. 31 (2005), 309-354.

[Chap] T. A. Chapman, Lectures on Hilbert Cube Manifolds, Regional Conference Ser. in Math. 28, Amer. Math. Soc., Providence 1976.

[Dav] R. Daverman, Decompositions of Manifolds, Academic Press, Orlando 1986.

[DW] R. Daverman, J. Walsh, Čech homology characterizations of infinite dimensional manifolds, Amer. J. Math. 103 (1981), 411-435.

[En] R. Engelking, Theory of Dimensions: Finite and Infinite, Heldermann, Lemgo 1995.

[Gre] J. H. Gresham, A class of infinite-dimensional spaces. II. An extension theorem and the theory of retracts, Fund. Math. 107 (1980), 237-245.

[Hat] A. Hatcher, Algebraic Topology, Cambridge Univ. Press, Cambridge 2002.

[Rad] T. Radul, On the classification of sigma hereditarily disconnected spaces, Mat. Stud., to appear.

[Sin] S. Singh, Exotic ANR's via null decompositions of Hilbert cube manifolds, Fund. Math. 125 (1985), 175-183.

[Sc] E. V. Shchepin, On Tikhonov manifolds, Dokl. Akad. Nauk SSSR 246 (1979), 551-554 (in Russian); Engl. transl.: Soviet Math. Dokl. 20 (1979), 511-515.

[Spa] E. Spanier, Algebraic Topology, McGraw-Hill, New York 1966.

[Us] V. V. Uspenskij, A selection theorem for C-spaces, Topology Appl. 85 (1998), 351-374.

[We] J. West, Open problems in infinite-dimensional topology, in: Open Problems in Topology, eds. J. van Mill, G. M. Reed, North-Holland, Amsterdam 1990, 523-597. 